NASA Contractor Report 185302

$$
\begin{aligned}
& \Rightarrow 05 \% 5 \\
& 24 p
\end{aligned}
$$

\title{
Reduction of Thermal Stresses in Continuous Fiber Reinforced Metal Matrix Composites With Interface Layers
}

S. Jansson and F.A. Leckie

University of California

Santa Barbara, California

October 1990

Prepared for

Lewis Research Center

Under Grant NAG3-894

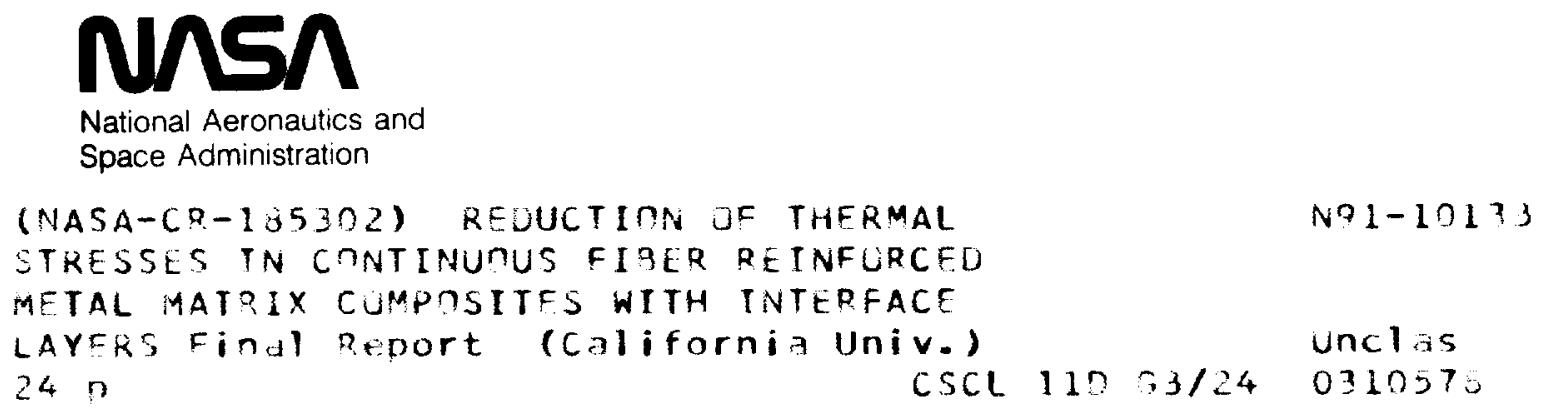





\section{INTRODUCTION}

Metal matrix composites reinforced with ceramic fibers have high strength and stiffness to density ratios and are attractive for many applications where weight is a concern. They are also expected to have good high temperature strength. Advantage can be taken of the dissimilar properties of fiber and matrix to create composites with superior properties: for example ceramic fibers provide high temperature strength and a ductile metal matrix provides energy dissipation. However, ceramics have a low coefficient of thermal expansion (CTE) and metals have a high CTE. The mismatch in CTE induces thermal stresses in the composite when subjected to temperature change. Time variations of temperature can reduce the fatigue life of metal matrix composite significantly [1]. Brittle intermetallic matrix materials, that are attractive because of low density, could crack during the fabrication of the composite at cool down after consolidation [2]. Such cracks could impair the performance of the composite dramatically.

Such problems have focused interest on the feasibility of using coatings on the fibers that would shield the matrix from the loading caused by the stiff fibers when the composite is subjected to a temperature change. Numerical parametric studies have been performed for continuous fiber reinforced composites by using concentric cylinder models [3] and by assuming the fibers are arranged in a square arrays [4]. The studies suggest that the layer should have a very low modulus and a CTE that is the average of the CTE's of the matrix and fiber in order to reduce the residual stresses in the matrix. However, the conclusions are based on limited numerical results and do not provide an understanding of the fundamental problem. It appears that the formulation of a simple model which includes all the essential features of the problem and can yield insight into the physics of the problem has been overlooked. The results of such a model are not expected to be exact but provide guidelines on where to focus the subsequent numerical studies. 
A simple model is formulated for a continuous fiber reinforced metal matrix composite with an interface layer when subjected to temperature change. The essential properties of an interface layer that could reduce the residual stresses in the matrix are identified. The possibility of improving the low cycle fatigue properties of the composite is also addressed.

\section{MODEL}

The composite consists of long aligned fibers with a thin interface layer in a metal matrix. The thermal loading of the composite is axi-symmetric with respect to the fibers and does not stimulate interaction between fibers in the transverse plane. The mechanical behavior of the composite may then be assumed to be given by a concentric cylinder model, Fig. 1. The composite is not subjected to transverse loading and the outer surface of the compound cylinder is traction free. The fibers are long and the stress and strain distributions are constant in the $z$-direction except at the end regions which are not studied here. Ceramic fibers are usually four to five times stiffer than metal matrix materials. Hence it is assumed, in order to simplify the calculations, that

$$
\frac{E_{f}}{E_{m}}>1
$$

and

$$
\frac{E_{f}}{E_{l}} \gg 1
$$

where the subscript $f$ denotes fiber, $m$ matrix, and $l$ interface layer. The fiber volume fraction, $c_{f}$, is assumed to be of the same order as the matrix volume fraction so that 


$$
\frac{c_{f}}{1-c_{f}}=1
$$

The radii defining the cylinder model (Fig.1) are related to the fiber volume fraction by the relation

$$
[\mathrm{a} / \mathrm{b}]^{2}=\mathrm{c}_{\mathrm{f}}
$$

The cylinder is assumed to be stress free at a given temperature $T_{1}$ and is then subjected to a homogeneous temperature change to a lower temperature $T_{2}$. The temperature change is defined as

$$
\Delta \mathrm{T}=\mathrm{T}_{1}-\mathrm{T}_{2}
$$

For the given assumptions the stiff fiber controls the thermal expansion in the axial direction of the cylinder model. Hence, the stress distribution in the matrix and interface layer is governed by a plane strain problem with a fixed inner radius at the fiber interface. The problem is formulated in terms of the differential CTE's of matrix and fiber

$$
\Delta \alpha_{\mathrm{m}}=\alpha_{\mathrm{m}}-\alpha_{\mathrm{f}}
$$

and of interface layer and fiber

$$
\Delta \alpha_{l}=\alpha_{l}-\alpha_{f}
$$

The stress distribution in the matrix is given by the plane strain solution for a thick walled cylinder subjected to an unknown internal pressıre p, a traction free outer surface, 
and a temperature change $\Delta \mathrm{T}$. Superimposing the solutions given in [5] for an internal pressure and a temperature change gives the stress distributions in the matrix

$$
\begin{gathered}
\sigma_{\mathrm{rm}}=-\mathrm{p} \frac{[\mathrm{b} / \mathrm{r}]^{2}-1}{[\mathrm{~b} / \mathrm{a}]^{2}-1} \\
\sigma_{\varphi \mathrm{m}}=\mathrm{p} \frac{[\mathrm{b} / \mathrm{r}]^{2}+1}{[\mathrm{~b} / \mathrm{a}]^{2}-1} \\
\sigma_{\mathrm{zm}}=\mathrm{p} \frac{2 v_{m}}{[\mathrm{~b} / \mathrm{a}]^{2}-1}+\mathrm{E}_{\mathrm{m}} \Delta a_{\mathrm{m}} \Delta \mathrm{T}
\end{gathered}
$$

The hoop strain is

$$
\begin{gathered}
\varepsilon_{\varphi \mathrm{m}}=\sum_{\mathrm{m}}^{\mathrm{p}} \frac{1-v_{\mathrm{m}}^{2}}{[\mathrm{~b} / \mathrm{a}]^{2}-1}\left[[\mathrm{~b} / \mathrm{r}]^{2}+1+\frac{v_{\mathrm{m}}}{1-v_{\mathrm{m}}}\left\{[\mathrm{b} / \mathrm{r}]^{2}-1\right\}\right] \\
-\left[1+v_{\mathrm{m}}\right] \Delta \alpha_{\mathrm{m}} \Delta \mathrm{T}
\end{gathered}
$$

The interface layer is assumed to be sufficiently thin for the variation of stress in the radial direction to be neglected. Because of the rigidity of the fiber the deformation of the layer is suppressed in the hoop and axial direction by the fiber so that

$$
\varepsilon_{\varphi l}=\varepsilon_{\mathrm{zl}}=0
$$

The layer is subjected to a pressure in the radial direction resulting from the contact with the matrix 


$$
\sigma_{\mathrm{r} l}=-\mathrm{p}
$$

The temperature change $\Delta \mathrm{T}$ introduces stresses in the hoop and longitudinal directions due to the constraint from the fibers (9). The stresses in the hoop and longitudinal direction are equal and are the sum of the stresses caused by the pressure and the thermal expansion

$$
\sigma_{\varphi l}=\sigma_{z l}=-\frac{v_{l}}{\Gamma-v_{l}} \mathrm{p}+\frac{1}{\Gamma-v_{l}} \mathrm{E}_{l} \Delta \alpha, \Delta \mathrm{T}
$$

The stresses in the layer give rise to the radial strain

$$
\varepsilon_{\mathrm{r} l}=-\left[1-\frac{2 v_{l}^{2}}{1-v_{l}}\right] \frac{\mathrm{p}}{\mathrm{E}_{l}}-\frac{1+v_{l}}{1-v_{l}} \Delta \alpha_{l} \Delta \mathrm{T}
$$

Compatibility in the radial displacement at the boundary between the inner surface of the matrix and the interface layer at $r=a$, requires

$$
\varepsilon_{\varphi \mathrm{m}} \mathrm{a}=\varepsilon_{\mathrm{rl}} \mathrm{t}
$$

The pressure in the radial direction at the layer is now derived by substituting (4) and (8) in (12) and using (11) to give

$$
\mathrm{p}=\frac{\left[1+v_{\mathrm{m}}\right]-\frac{\mathrm{t} \Delta \alpha_{l}}{\mathrm{a} \Delta \alpha_{\mathrm{m}}} \frac{1+v_{l}}{1-v_{l}}}{\left[1-v_{\mathrm{m}}^{2}\right]\left[\frac{1+\mathrm{c}_{\mathrm{f}}}{1-\mathrm{c}_{\mathrm{f}}}+\frac{v_{\mathrm{m}}}{1-v_{\mathrm{m}}}\right]+\frac{\mathrm{t} \mathrm{E}_{\mathrm{m}}}{\mathrm{a} \mathrm{E}_{l}} \frac{v_{l}}{1-v_{l}}} \mathrm{E}_{\mathrm{m}} \Delta \alpha_{\mathrm{m}} \Delta \mathrm{T}
$$




\section{STRESSES IN MATRIX}

The highest stresses in the matrix occur at the inner surface of the cylinder, $r=a$. The principal stresses are given by $(7 a-c)$ by letting $r=a$ and using (4). The $v$. Mises effective stress

$$
\sigma^{e}=\sqrt{\sigma_{r}^{2}+\sigma_{\varphi}^{2}+\sigma_{z}^{2}-\sigma_{r} \sigma_{\varphi}-\sigma_{r} \sigma_{z}-\sigma_{\varphi} \sigma_{z}}
$$

can be calculated from the principal stresses given in (7) as

$$
\begin{gathered}
\sigma_{m}^{e}=\left[\left[p \frac{c_{f}}{1-c_{f}}\right]^{2}\left[3 / c_{f}^{2}+1-4 v_{m}\left(1-v_{m}\right)\right]-p E_{m} \Delta \alpha_{m} \Delta T \frac{c_{f}}{1-c_{f}} 2\left[1-2 v_{m}\right]+\right. \\
\left.\left[E_{m} \Delta \alpha_{m} \Delta T\right]^{2}\right]^{1 / 2}
\end{gathered}
$$

The stresses in the matrix are readily found by first calculating the interface layer pressure for a given temperature change $\Delta \mathrm{T}$ using (13) and using this value in $(7 \mathrm{a}-\mathrm{c})$ and (15) to determine the stresses. The term $E_{m} \Delta \alpha_{m} \Delta T$ enters in all the expressions for stress and all results can be normalized with respect to this term. The stresses at the inner radius of the matrix are shown in Fig. 2 as a function of normalized pressure for a fiber volume fraction $c_{\mathrm{f}}=0.4$ in a $\mathrm{Ti}_{3} \mathrm{Al}$ matrix for which $v_{\mathrm{m}}=0.25$. It can be seen that pressure at the interface causes increased tensile stresses in the hoop and axial direction which could result in matrix cracking, which is initiated from defects at the fiber matrix interface. The effective stress is a minimum when the interface pressure that is close to zero. Consequently the pressure should be low in order to have good low cycle fatigue properties of the composite. 
For any given composite a plot similar to Fig. 2 can be produced. The value of the critical stress for matrix cracking,$\sigma_{\mathrm{cm}}$, sets an upper limit for the principal stresses $\sigma_{\varphi m}$ , $\sigma_{\mathrm{zm}}$, and $\sigma_{\mathrm{rm}}$ and defines bounds on the pressure $\mathrm{p}$ if matrix failure is to be avoided. The allowable effective stress,$\sigma_{\mathrm{ym}}$, in the matrix defines other bounds on the pressure. These bounds define a window for the interface pressure (Fig 2.) if the failure criteria are not to be violated. If the calculated pressure does not fall within the allowable window it must be adjusted to avoid matrix cracking or failure due to high effective stress.

Inspection of (13) reveals that the pressure may be reduced in two ways by the introduction of interface layers. A compliant layer with low modulus would increase the denominator in (13) while a compensating layer with a high coefficient of thermal expansion would decrease the numerator in (13). An appreciation for the potential of the two effects can be obtained by evaluating the requirements to reduce the pressure by a factor two. To achieve this condition using a compliant layer means that the two terms in the denominator of (13) are equal and gives the result,

$$
\frac{E_{l}}{E_{m}}=\frac{t}{a} \frac{v_{l}\left(1-c_{f}\right)}{\left(1-v_{l}\right)\left(1+v_{m}\right)\left[\left(1-v_{m}\right)\left(1+c_{f}\right)+v_{m}\left(1-c_{f}\right)\right]}
$$

For the present composite, using $v_{l}=0.3$, this requires that

$$
\frac{E_{l}}{E_{m}}=0.17 \frac{t}{a}
$$

In general the layer has to be thin in order to keep the density down of the composite, and a realistic value of $t / a$ is 0.1 . This requires that the modulus of the layer must be unrealistically low for the pressure to be reduced by a factor two. Hence it is deduced that a compliant layer is not an efficient way to reduce thermal stresses in the matrix. 
Furthermore, the second term in the denominator of (13) is usually sufficiently small to be neglected in the calculations and the pressure is then linearly dependent on the normalized thermal expansion of the layer, $\Delta \alpha_{l} l / \Delta \alpha_{\mathrm{m}}$ a. This is demonstrated in Fig. 3 where it can be seen that the influence of $E_{m} / E_{\rho}^{a}$ is small.

A reduction of the pressure by a factor two with a compensating layer requires that the second term in the numerator of (13) is equal to half of the first term, to give,

$$
\frac{\Delta \alpha_{l}}{\Delta \alpha_{\mathrm{m}}}=\frac{\mathrm{a}}{\mathrm{t}} 1-\mathrm{v}_{l} 1+\mathrm{v}_{\mathrm{m}} \frac{1+\mathrm{v}_{l}}{2}
$$

For the present composite this gives the condition

$$
\frac{\Delta \alpha_{l}}{\Delta \alpha_{m}}=0.34 \frac{a}{t}
$$

Hence when $t / a=0.1$ the differential CTE for the layer is $\Delta \alpha_{l}=3.4 \Delta \alpha_{\mathrm{m}}$. For the case of $\operatorname{SiC}$ fibers $\left(\alpha_{\mathrm{f}} \approx 510^{-6} 1 / \mathrm{C}\right)$ in a $\operatorname{Ti}_{3} \mathrm{Al}$ matrix $\left(\alpha_{\mathrm{m}} \approx 1010^{-6}\right)$ requires that $\alpha_{l} \approx 2010^{-6}$ if the pressure is to be reduced by a factor two. Handbook values [6] of metals with a reasonably high melting point and high CTE are: silver $2510^{-6}$, copper $1710^{-6}$, and manganese $2210^{-6}$. It is evident therefore that an interface layer of readily available materials with high CTE has the potential to substantially reduce thermal stresses in the matrix.

The results given in Fig. 2 and Fig. 3 have been combined in Fig. 4 where the highest principal stress and effective stress at the inner radius of the matrix cylinder are plotted against the normalized thermal expansion of the layer. No interface layer corresponds to $\Delta \alpha_{l} t \Delta \alpha_{\mathrm{m}} \mathrm{a}=0$. It can be deduced that a layer with a lower CTE than the 
matrix will increase the stresses in the matrix and a layer with a higher CTE than the matrix reduces the stresses in the matrix. The effective and principal stress have minima for different layer characteristics and the required thermal expansion of the layer, $\Delta \alpha_{l} t$, has to be a compromise as discussed earlier. The highest effective stress in the matrix, (15), governs the low cycle fatigue performance of the composite when it is subjected to thermal cycling. Shake down to linear elastic stress strain response is expected when the cyclic effective stress, based on a linear elastic calculation, is less than twice the yield stress. The shake down condition is plotted in Fig. 5 as a function of the normalized thermal expansion of the layer. It can be seen that an interface layer can double the allowable temperature range for elastic shake down in the matrix.

\section{STRESSES IN INTERFACE LAYER}

The tradeoff for the improved stress state in the matrix is that the thermal mismatch has to be taken up by the interface layer. The deformation of the layer is constrained in the hoop and longitudinal direction so that tensile stresses develop in these directions. The highest principal stress in the interface layer which is given by substituting (13) in (10b), is shown in Fig. 6 as a function of the dimensionless parameters defining the problem. The stress is strongly dependent on the layer modulus, $\mathrm{E}_{l} / \mathrm{E}_{\mathrm{m}}$, and CTE, $\Delta \alpha_{l} / \Delta \alpha_{\mathrm{m}}$, but weakly dependent on the layer thickness $t / a$. This indicates that the stress is dominated by the constrained thermal expansion [ given by the second term in (10b)] and is weakly

dependent on the interface pressure [ given by the first term in (10b)]. The product $\Delta \alpha_{l} t$ governs the reduction of stress in the matrix (Fig. 4) and must have a required value to reduce the stresses in the matrix to an acceptable level. It can be deduced from Fig. 4 and Fig. 6 that it is more favorable to have a thick layer and a moderate high layer CTE than a thin layer and a high layer CTE, to meet the requirements of stress reduction in the matrix and avoidance of high tensile stresses in the layer. 
The effective stress in the layer is given by inserting $(10 a-c)$ in (14) to give

$$
\sigma_{1}^{e}=\frac{1}{1-v_{l}}\left|E_{l} \Delta \alpha, \Delta T+\left(1-2 v_{l}\right) p\right|
$$

Shake down to elastic response in the layer is expected when the cyclic part of the effective stress is less than twice the yield stress of the layer. The shake down limit has been plotted in a normalized form in Fig. 7 for different layer modulus and thickness. The stress in the layer is strongly dependent on the layer modulus and to ensure shake down it is desirable to have a layer with a high yield stress and a low modulus.

\section{THE EFFECT OF YIELDING OF THE INTERFACE LAYER}

The previous calculations indicate that the layer can be subjected to high stresses and may yield. An estimation of how yielding in the layer affects reduction of the stresses in the matrix can be obtained by examining the behavior of an elastic-perfectly plastic layer. In the analyses the layer is assumed to be in an initial elastic stress state which satisfies the yield condition (14). The cylinder is subsequently subjected to a further temperature change $\mathrm{dT}$ so that the radial pressure changes by $\mathrm{dp}$. The loading is such that the layer remains yielded during the subsequent deformation requiring the effective stress to be constant. Differentiation of (14) and using the condition at yield, $(10 \mathrm{~b}, \mathrm{c})$, gives an incremental stress change in the layer which is hydrostatic. Thus, the stress increments are

$$
\mathrm{d} \sigma_{\mathrm{zl}}=\mathrm{d} \sigma_{\varphi l}=\mathrm{d} \sigma_{\mathrm{rl}}=-\mathrm{dp}
$$


Since the total strains of the layer in the $\varphi$ and $z$ directions are constrained to be zero, $(9 a, b)$, it follows that the plastic strains in these directions are the sum of the elastic and thermal part

$$
\mathrm{d} \varepsilon_{\varphi l}^{\mathrm{p}}=\mathrm{d} \varepsilon_{\mathrm{zl}}^{\mathrm{p}}=-\Delta \alpha, \mathrm{dT}+\frac{1-2 v_{1}}{\mathrm{E}_{1}} \mathrm{dp}
$$

The plastic deformation is incompressible requiring

$$
\mathrm{d} \varepsilon_{\mathrm{r} l}^{\mathrm{p}}=-\mathrm{d} \varepsilon_{\varphi l}^{\mathrm{p}}-\mathrm{d} \varepsilon_{\mathrm{z} l}^{\mathrm{p}}
$$

and the total strain increment in the radial direction is the sum of the elastic, thermal, and plastic component and becomes

$$
\mathrm{d} \varepsilon_{\mathrm{r} l}^{\mathrm{Pl}}=\frac{1-2 \mathrm{v}_{l}}{\mathrm{E}_{l}} \mathrm{dp}+\Delta \alpha \mathrm{dT}+\mathrm{d} \varepsilon_{z_{l}}^{\mathrm{p}}=3 \Delta \alpha \mathrm{dT}-3 \frac{1-2 \mathrm{v}_{l}}{\mathrm{E}_{l}} \mathrm{dp}
$$

The radial strain in the layer governs stress reduction in the matrix and an appreciation for the effect of the yielded layer can be determined by comparing the radial strain for an elastic layer, Eq. (11), with the radial strain for an elasto-plastic layer, Eq. (22). The contribution from the pressure $p$ in both these equations is small and can be neglected. The strains are then only linearly dependent on the temperature change. By letting $\Delta T=$ $-d T$ in Eq. (11) the ratio of the two strains is found to be

$$
\frac{\mathrm{d} \varepsilon_{\mathrm{r} l}^{\mathrm{Pl}}}{\mathrm{d} \varepsilon_{\mathrm{rl}}^{\mathrm{el}}}=\frac{3\left(1-v_{l}\right)}{1+v_{l}}
$$


To estimate the effect of the elasto-plastic deformation, the second term in the numerator of (13) must be multiplied by the right hand side of (22) when calculating the pressure. For the present data, with $v_{l}=0.3$, this implies that the thermal expansion of the layer becomes 1.5 times more effective in reducing the pressure. Hence the results of the elastic solutions given in Fig. 3-5 can be readily modified to include plastic deformation in the layer.

\section{CONCLUSIONS}

A compliant layer must to be unrealistically flexible to reduce thermal stresses in the matrix.

A compensating layer with a high coefficient of thermal expansion (not unrealistically high) has the potential of reducing the thermal stresses in the matrix significantly.

The hoop stress in the matrix close to the fiber can be reduced substantially but the axial stress in the matrix is less affected by a layer. This implies that compensating layers can be expected to be successful in preventing cracking in composites where predominantly radial cracking is observed in the matrix.

The shake down range to linear elastic response in the matrix can be extended significantly with the addition of a compensating layer.

The thermal mismatch between fiber and matrix is taken up by the layer which can cause high stresses in the layer. The required stress reduction in the matrix sets a requirement on the value of the thermal expansion of the layer, $\Delta \alpha_{l} t$. However, the stress 
in the layer is proportional to $\Delta \alpha_{l}$ so that a high layer CTE can cause high stress in the layer. In order to have low stress in the layer it is more favorable to have a moderately high layer CTE and thick layer than a thin layer with very high layer CTE. A low elastic modulus of the layer reduces the stresses in the layer. The effect of plastic deformation of the layer on the stress reduction in the matrix is equivalent to increasing the CTE of the layer by 1.5 .

Thermal stresses build up in the layer because the required thermal expansion in the radial direction is different from the thermal expansions in the hoop and longitudinal directions that are governed by the fiber. The stresses in the layer could be minimized if a layer could be developed with an anisotropic thermal expansion such that the thermal expansion is close to the fiber CTE in the longitudinal and hoop directions and high in the radial direction.

Residual stresses can be reduced by Compensating layers for other reinforcements shapes than fibers. Thermal expansion of reinforcements with lower aspect ratios is less anisotropic and can be better compensated for by a layer with an isotropic CTE. The thermal mismatch for a spherical inclusion can be compensated for fully and no residual stresses would develop in the matrix.

\section{ACKNOWLEDGEMENT}

The work was supported by a grant from the NASA Lewis Research Center. The authors wish to express their gratitude to Dr. Steve Arnold for the encouragement and support. 


\section{REFERENCES}

[1] Castelli, M. G. et. al., "Development of Thermomechanical Testing Techniques for Advanced Composites," 2nd HITEMP Review, NASA CP-10039, paper 43, 1989.

[2] Brindley, P. K., Bartolotta, P. A. and MacKay, R. A., "Thermal and Mechanical Fatigue of $\mathrm{SiC} / \mathrm{Ti}_{3} \mathrm{Al}+\mathrm{Nb}$," 2nd HITEMP Review, NASA CP-10039, paper 52, 1989.

[3] Ghosn, L. J. and Bradley, A. L. "Optimum Interface Properties for Metal Matrix Composites," NASA TM-102295. 1989

[4] Caruso, J. J., Chamis, C. C. and Brown, H. C., "Parametric Studies to Determine the Effects of Compliant Layers on Metal Matrix Composite Systems," NASA TM-102465, 1990.

[5] Timoshenko, S. P. and Goodier, J. N., "Theory of Elasticity," McGraw-Hill, Auckland, 1970.

[6] Boyer, h. E. and Gall, T. L., "Metals Handbook, Desk Edition,", ASM, Metals Park, 1985. 


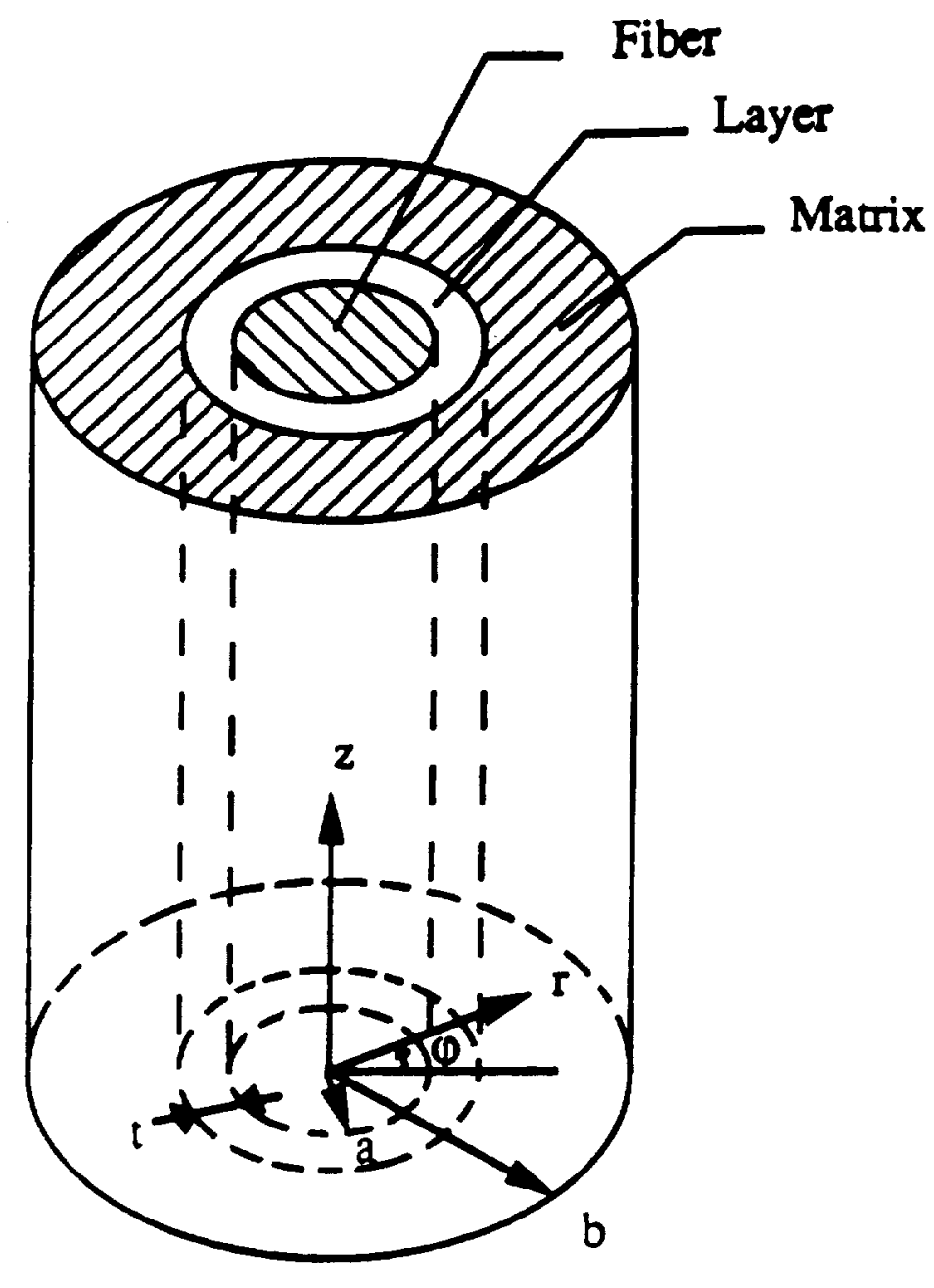

Fig. 1 Concentric cylinder model of composite with a thin interface layer. 


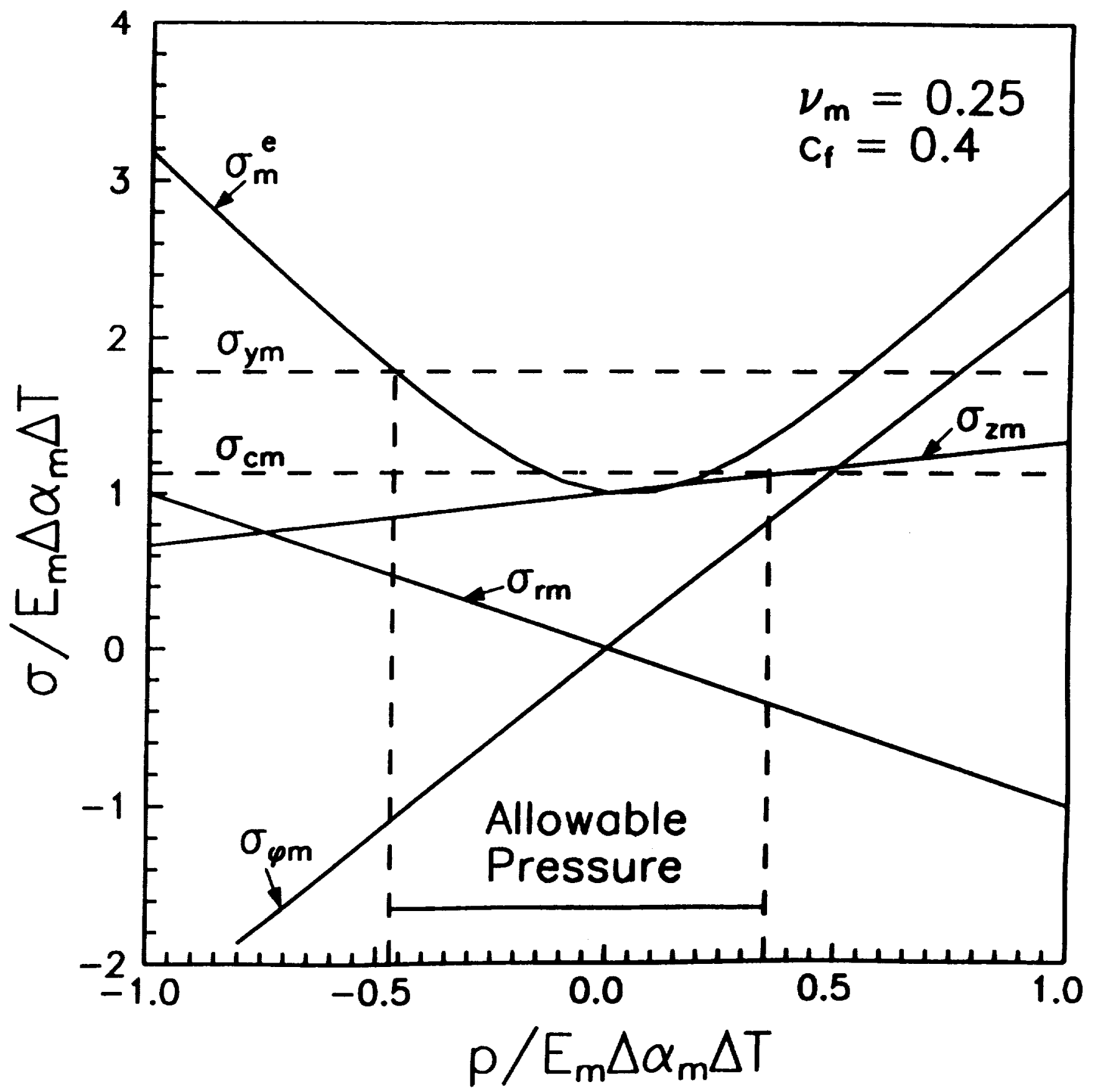

Fig. 2 Stresses at the inner surface of the matrix cylinder as a function of normalized pressure at the matrix fiber interface. 


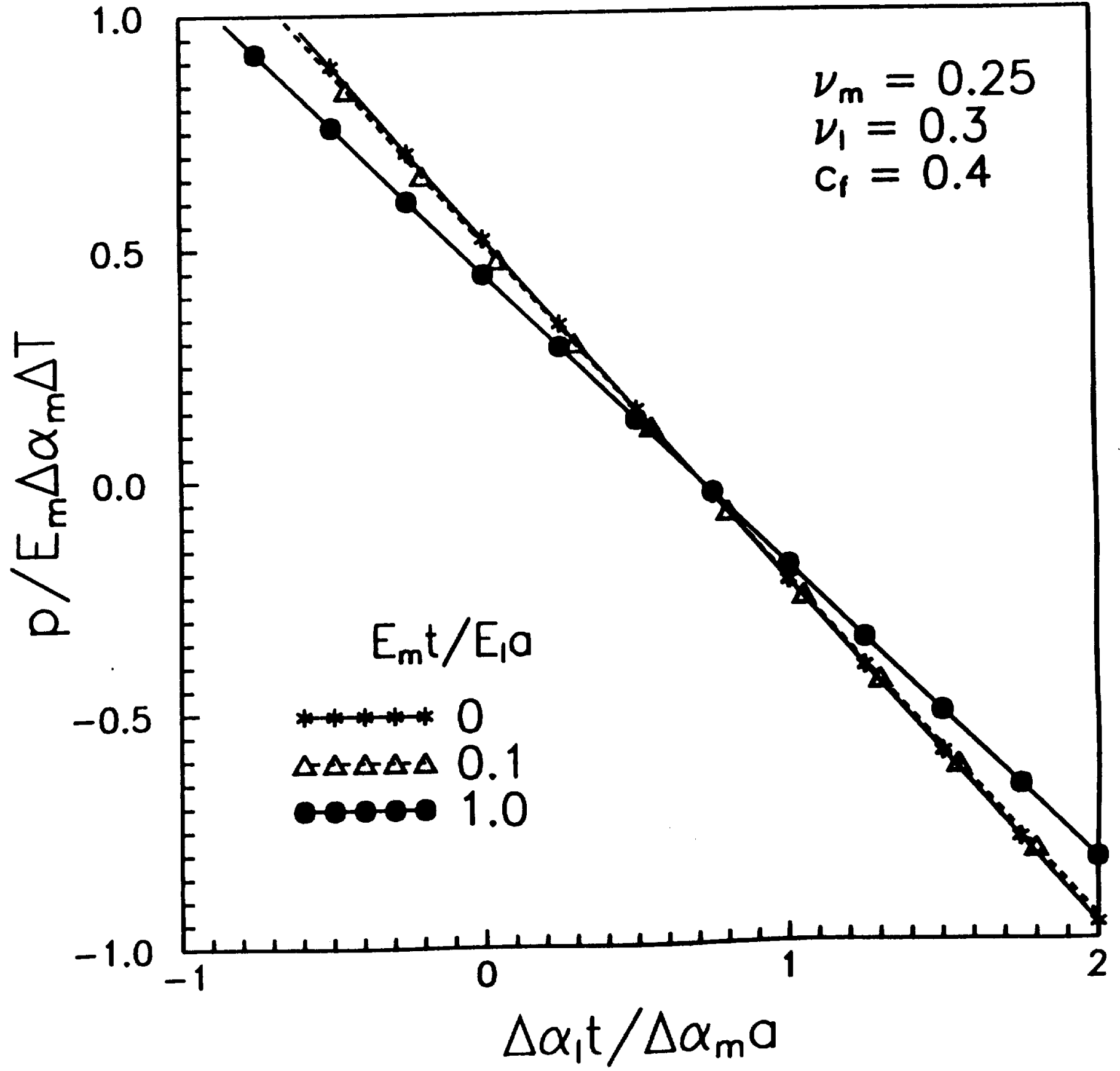

Fig. 3 Relation between normalized pressure at matrix fiber interface and relative coefficients of thermal expansion and layer thickness. 


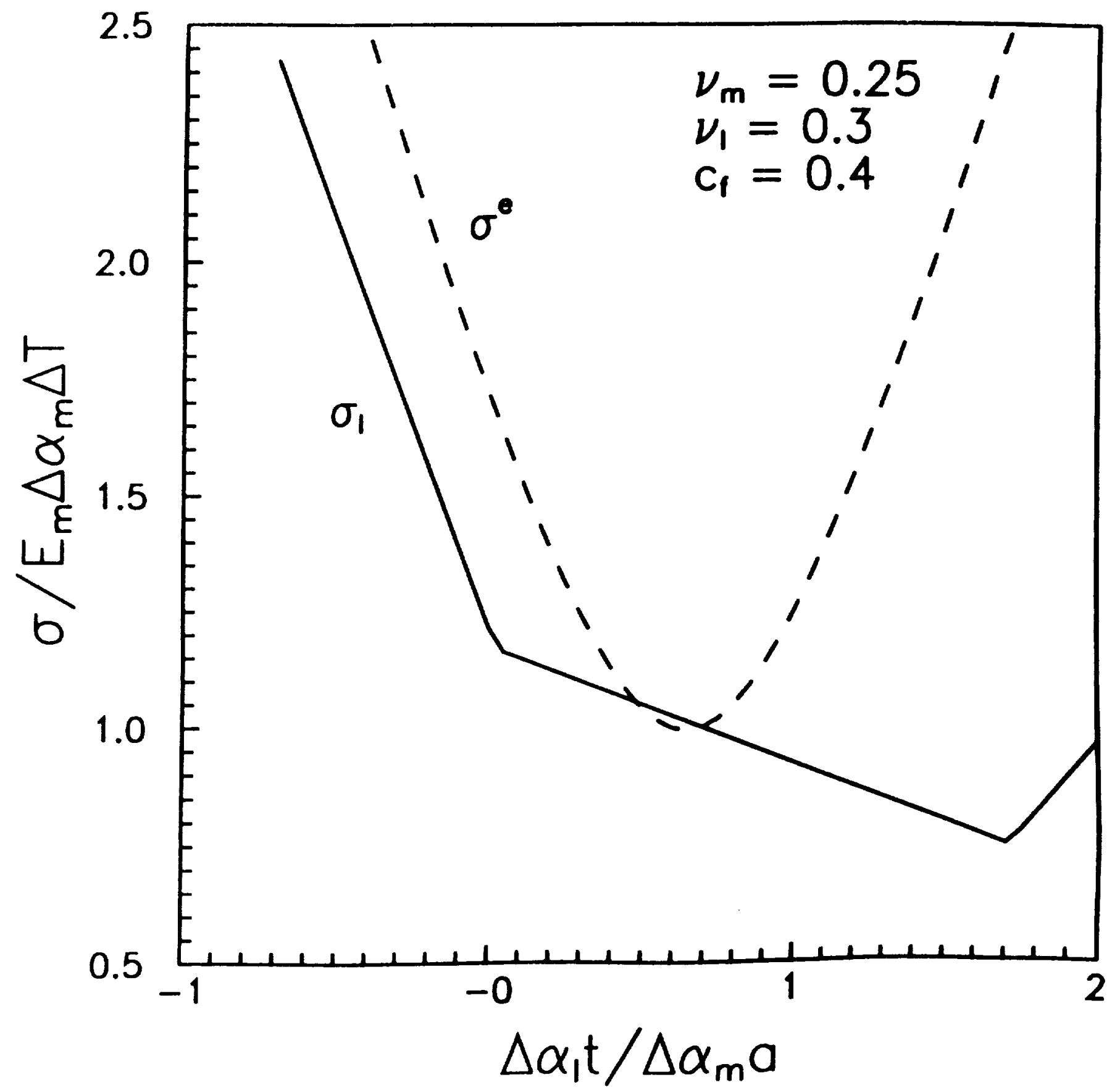

Fig. 4 Maximum principal stress and effective stress at the inner surface of the matrix cylinder as a function of the relative thermal expansion of the layer. 


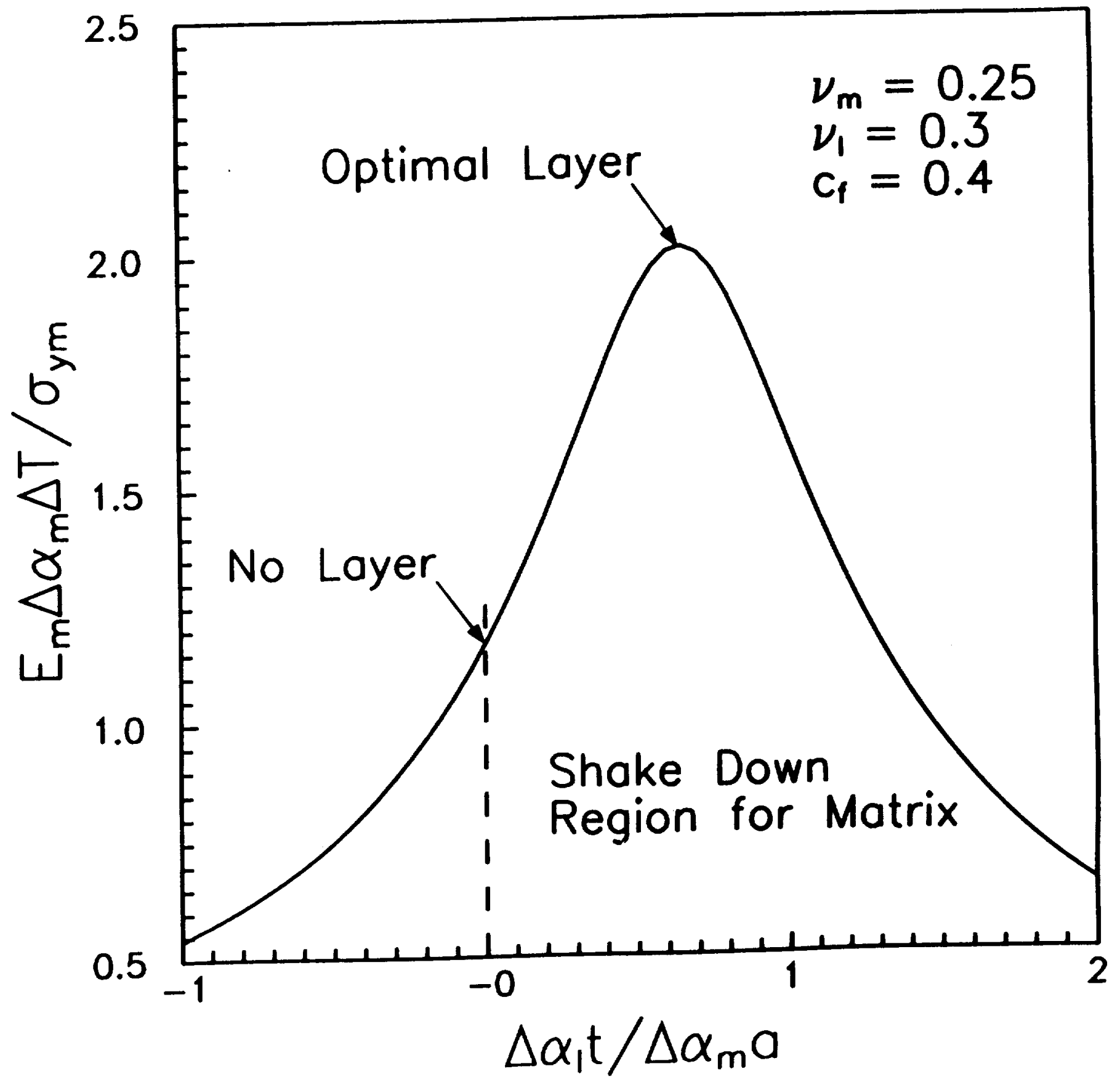

Fig. 5 Condition for elastic shake down in the matrix. 


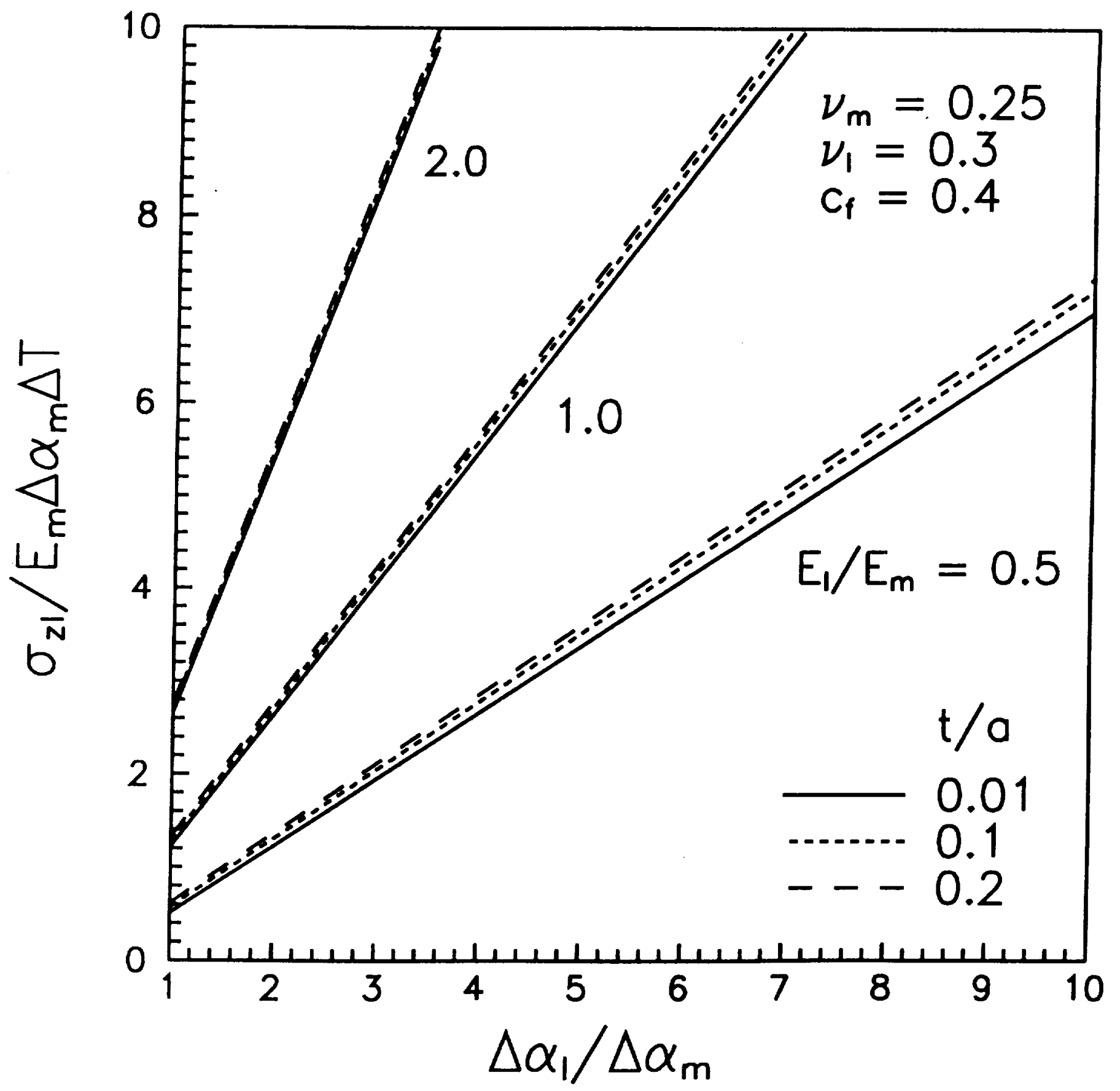

Fig. 6 Longitudinal and hoop tensile stress in interface layer. 


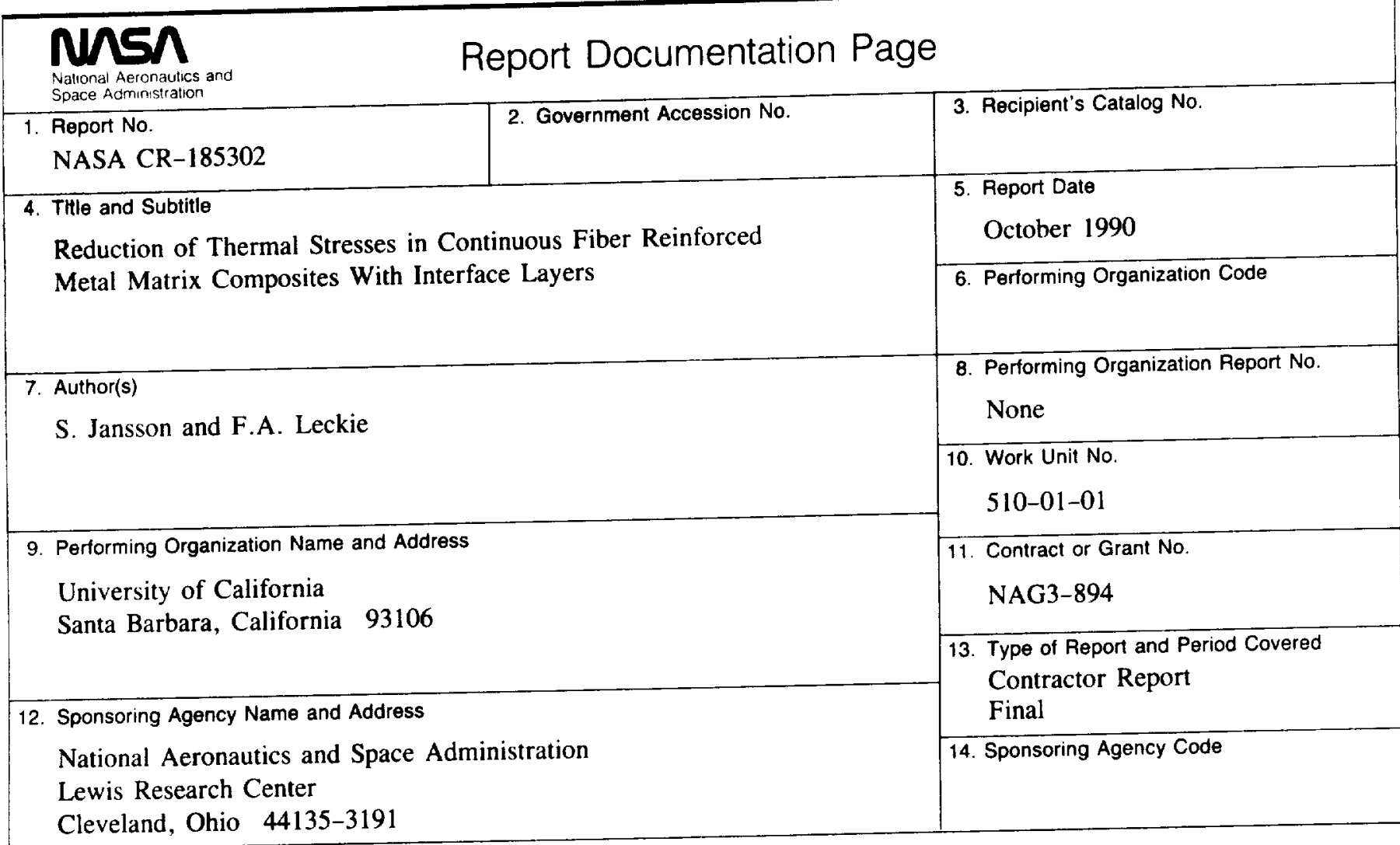

15. Supplementary Notes

Project Manager, Steven M. Arnold, Structures Division, NASA Lewis Research Center.

16. Abstract

The potential of using an interface layer to reduce thermal stresses in the matrix of composites with a mismatch in coefficients of thermal expansion of fiber and matrix has been investigated. It was found that compliant layers, with properties of readily available materials, do not have the potential to reduce thermal stresses significantly. However, interface layers with high coefficient of thermal expansion can compensate for the mismatch and reduce thermal stresses in the matrix significantly.

17. Key Words (Suggested by Author(s))

Composites; Metallic; Thermal mismatch;

Shake down; Compliant layer
18. Distribution Statement

Unclassified - Unlimited

Subject Category 24
19. Security Classif. (of this report)

Unclassified
20. Security Classif. (of this page)

Unclassified

21. No. of pages

22
22. Price* 


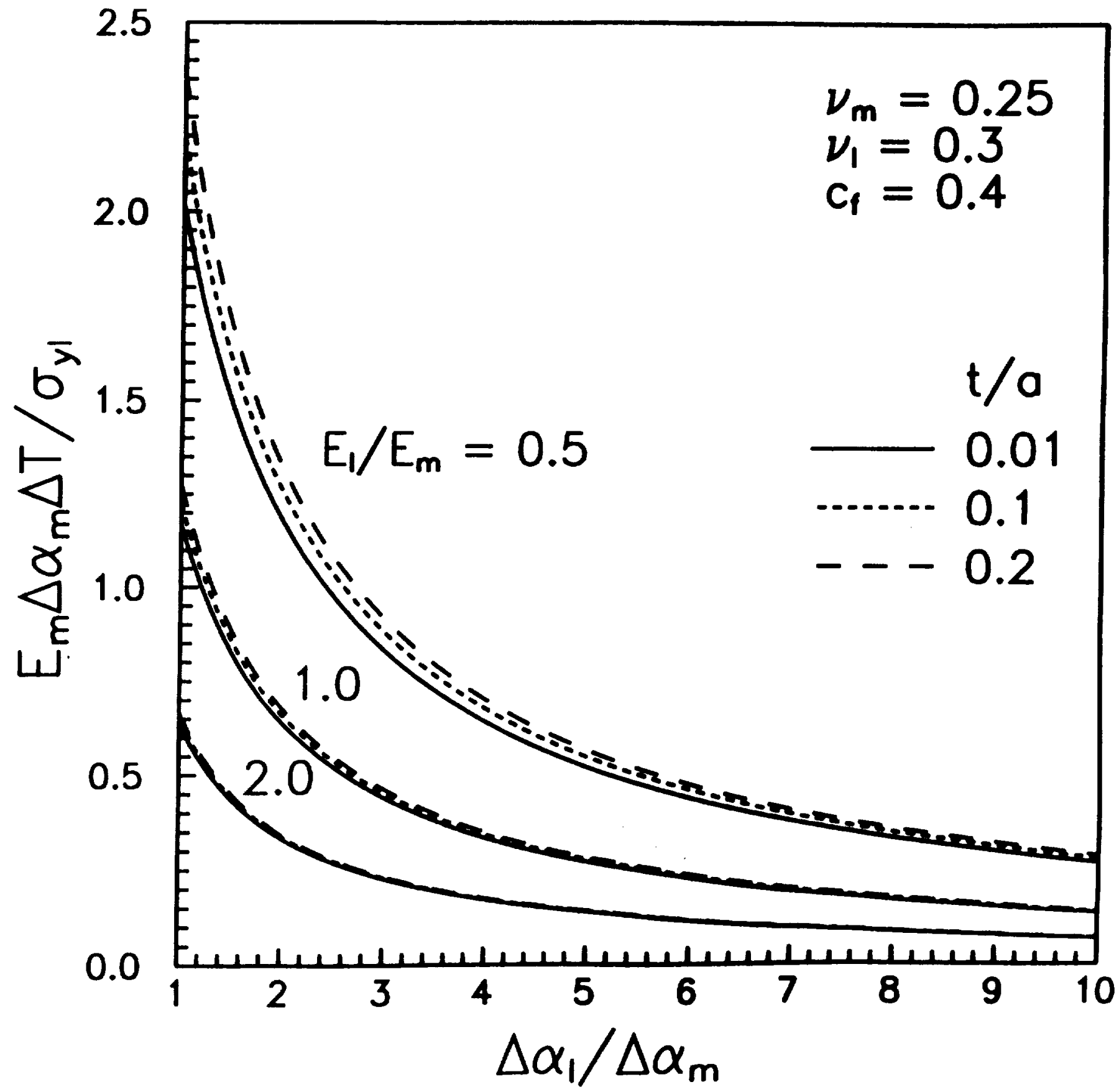

Fig. 7 Condition for elastic shake down in the interface layer. Shake down occurs for temperature changes corresponding states under the curve defined by the composite parameters. 
National Aeronautics and

Space Administration

Lewis Research Center

Cleveland. Ohio 44135

Oriated Buminas

Penally lor Prived uno $\$ 000$
FOUFTH CLASS MAII

ADDRESS CORRECTION REQUESTED

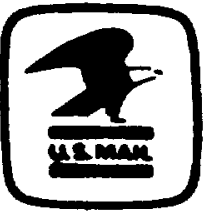

Poetage and Foes Paid Nationd Aoronautics and Soece Administration NASA-15i 